\title{
Some aspects of quantum sufficiency for finite and full von Neumann algebras
}

\author{
Andrzej Łuczak ${ }^{1}$
}

Received: 5 November 2019 / Revised: 25 May 2021 / Accepted: 20 June 2021 /

Published online: 14 July 2021

(c) The Author(s) 2021

\begin{abstract}
Some features of the notion of sufficiency in quantum statistics are investigated. Three kinds of this notion are considered: plain sufficiency (called simply: sufficiency), strong sufficiency and Umegaki's sufficiency. It is shown that for a finite von Neumann algebra with a faithful family of normal states the minimal sufficient von Neumann subalgebra is sufficient in Umegaki's sense. Moreover, a proper version of the factorization theorem of Jenčová and Petz is obtained. The structure of the minimal sufficient subalgebra is described in the case of pure states on the full algebra of all bounded linear operators on a Hilbert space.
\end{abstract}

Keywords Quantum sufficiency · Von Neumann algebra · Conditional expectation · Normal and pure states

Mathematics Subject Classification Primary 46L53; Secondary 62B05

\section{Introduction}

Let $\mathcal{M}$ be a von Neumann algebra, let $\mathcal{N}$ be its von Neumann subalgebra, and let $\left\{\rho_{\theta}: \theta \in \Theta\right\}$ be a family of normal states on $\mathcal{M}$. In [4] the notion of strong sufficiency of the subalgebra $\mathcal{N}$ for the family $\left\{\rho_{\theta}: \theta \in \Theta\right\}$ was introduced (and further investigated in [5]) as a generalisation of sufficiency in Umegaki's sense considered earlier in $[13,14]$. In this operator algebra setup, strong sufficiency of $\mathcal{N}$ means the existence of a normal two-positive map $\alpha: \mathcal{M} \rightarrow \mathcal{N}$ such that

$$
\rho_{\theta} \circ \alpha=\rho_{\theta}, \quad \theta \in \Theta
$$

$凶$ Andrzej Łuczak

andrzej.luczak@wmii.uni.lodz.pl

1 Faculty of Mathematics and Computer Science, Łódź University, ul. S. Banacha 22, 90-238 Łódź, Poland 
If the map $\alpha$ is a normal conditional expectation onto $\mathcal{N}$, then we get sufficiency in Umegaki's sense. An obvious and natural generalisation of the notion of strong sufficiency would consist in giving up the, rather technical, requirement of two-positivity and replacing it by positivity. Some work in this setup was done in [6], and in the present paper, we continue this line of investigation. As was pointed out in [6], this approach is additionally motivated by considerations from quantum hypothesis testing theory, where a different notion of sufficiency is employed, namely, the sufficiency of $\mathcal{N}$ means that we can find there an optimal measurement minimising the Bayes risk (cf. [7]).

In the first part of the paper, we show that for a $\sigma$-finite finite von Neumann algebra with a faithful family of normal states the minimal sufficient von Neumann subalgebra is sufficient in Umegaki's sense, and obtain a proper version of the factorization theorem due to Jenčová and Petz. In the second part, we are dealing with the full algebra $\mathbb{B}(\mathcal{H})$ and a family of pure states. There we give a description of the minimal sufficient subalgebra together with an explicit form of the conditional expectation defining this sufficiency.

\section{Preliminaries and notation}

Let $\mathcal{H}$ be a Hilbert space with a scalar product $\langle\cdot \mid \cdot\rangle$ and norm $\|\cdot\|$, and let $\mathcal{M}$ be an arbitrary von Neumann algebra of operators acting on $\mathcal{H}$, with identity $\mathbb{1}$, and predual $\mathcal{M}_{*}$. $\mathcal{M}$ is said to be $\sigma$-finite (or countably decomposable) if any family of pairwise orthogonal projections in $\mathcal{M}$ is countable. In our considerations, we restrict attention to such algebras.

A state on $\mathcal{M}$ is a bounded positive linear functional $\rho: \mathcal{M} \rightarrow \mathbb{C}$ of norm one. A state $\rho$ is said to be normal if it is continuous in the $\sigma$-weak topology on $\mathcal{M}$, in other words, $\rho \in \mathcal{M}_{*}$. For a normal state $\rho$, its support, denoted by $\mathrm{s}(\rho)$, is defined as the smallest projection $e$ in $\mathcal{M}$ such that $\rho(e)=\rho(\mathbb{1})$. In particular, we have

$$
\rho(\mathrm{s}(\rho) A)=\rho(A \mathrm{~s}(\rho))=\rho(A), \quad A \in \mathcal{M},
$$

and if $\rho(\mathrm{s}(\rho) A \mathrm{~s}(\rho))=0$ for $\mathrm{s}(\rho) A \mathrm{~s}(\rho) \geq 0$ then $\mathrm{s}(\rho) A \mathrm{~s}(\rho)=0$.

A normal state $\rho$ is said to be faithful if for each positive element $A \in \mathcal{M}$ from the equality $\rho(A)=0$ it follows that $A=0$. It is easily seen that the faithfulness of $\rho$ is equivalent to the relation $\mathrm{s}(\rho)=\mathbb{1}$. The existence of a normal faithful state on $\mathcal{M}$ is equivalent to $\mathcal{M}$ being $\sigma$-finite.

To each unit vector $\psi \in \mathcal{H}$ there corresponds a normal state, denoted by the same symbol and called a vector state, defined as

$$
\psi(A)=\langle\psi \mid A \psi\rangle, \quad A \in \mathcal{M} .
$$

By a slight abuse of language, we shall speak of the vector $\psi$ itself as a pure state. By $P_{[\psi]}$ shall be denoted the projection onto the space spanned by the vector $\psi$ (in Dirac notation, $\left.P_{[\psi]}=|\psi\rangle\langle\psi|\right)$. 
Let $\left\{\rho_{\theta}: \theta \in \Theta\right\}$ be a family of normal states on a von Neumann algebra $\mathcal{M}$. This family is said to be faithful if for each positive element $A \in \mathcal{M}$ from the equality $\rho_{\theta}(A)=0$ for all $\theta \in \Theta$ it follows that $A=0$. Similarly to the case of one state, it is seen that the faithfulness of the family is equivalent to the relation

$$
\bigvee_{\theta \in \Theta} \mathrm{s}\left(\rho_{\theta}\right)=\mathbb{1}
$$

In particular, for a family of pure states $\left\{\psi_{\theta}: \theta \in \Theta\right\}$, its faithfulness amounts to the equality $\overline{\operatorname{Lin}\left\{\psi_{\theta}: \theta \in \Theta\right\}}=\mathcal{H}$.

Let $P$ be a projection in a von Neumann algebra $\mathcal{M}$. A reduced von Neumann algebra $\mathcal{M}_{P}$ is defined as an algebra of operators acting on the Hilbert space $P(\mathcal{H})$ by the formula

$$
\mathcal{M}_{P}=\{P A \mid P(\mathcal{H}): A \in \mathcal{M}\} .
$$

Let $\left\{\mathcal{M}_{i}: i \in I\right\}$ be a family of von Neumann algebras acting on Hilbert spaces $\mathcal{H}_{i}$. The direct sum von Neumann algebra is defined as

$$
\sum_{i \in I}^{\oplus} \mathcal{M}_{i}=\left\{A=\left(A_{i}\right)_{i \in I}: A_{i} \in \mathcal{M}_{i} \text { for all } i \in I, \sup _{i \in I}\left\|A_{i}\right\|<\infty\right\},
$$

where the operators $A=\left(A_{i}\right)_{i \in I}$ act on the Hilbert space

$$
\mathcal{H}=\bigoplus_{i \in I} \mathcal{H}_{i}
$$

as

$$
A\left(\left(\xi_{i}\right)_{i \in I}\right)=\left(A_{i} \xi_{i}\right)_{i \in I}
$$

Let $\mathcal{M}$ and $\mathcal{N}$ be von Neumann algebras. A linear map $\alpha: \mathcal{M} \rightarrow \mathcal{N}$ is said to be normal if it is continuous in the $\sigma$-weak topologies on $\mathcal{M}$ and $\mathcal{N}$, respectively. It is called unital if $\alpha(\mathbb{1})=\mathbb{1}$.

The algebra of all bounded linear operators on $\mathcal{H}$ will be denoted by $\mathbb{B}(\mathcal{H})$. For arbitrary $\mathcal{T} \subset \mathbb{B}(\mathcal{H})$ by $\mathcal{T}_{h}$ will be denoted the set of hermitian elements of $\mathcal{T}$.

The Jordan product of two operators $A, B \in \mathbb{B}(\mathcal{H})$ will be denoted by the symbol 'o':

$$
A \circ B=\frac{1}{2}(A B+B A),
$$

and by a slight abuse of notation, the same symbol will be used for superpositions of a functional $\varphi$ on $\mathcal{M}$ and a map $\mathbb{E}$ on $\mathcal{M}: \varphi \circ \mathbb{E}$, as well as of two maps $\mathbb{E}, \mathbb{F}$ on $\mathcal{M}$ : $\mathbb{E} \circ \mathbb{F}$.

For arbitrary $\mathcal{T} \subset \mathbb{B}(\mathcal{H})$, by $W^{*}(\mathcal{T})$, we shall denote the von Neumann algebra generated by $\mathcal{T}$, i.e., the smallest von Neumann algebra containing $\mathcal{T}$. 
Let $\mathcal{M}$ be a von Neumann algebra, let $\left\{\rho_{\theta}: \theta \in \Theta\right\}$ be a family of normal states on $\mathcal{M}$, and let $\mathcal{N}$ be a von Neumann subalgebra of $\mathcal{M}$. $\mathcal{N}$ is said to be sufficient for the family of states $\left\{\rho_{\theta}: \theta \in \Theta\right\}$ if there exists a normal positive linear unital map $\alpha: \mathcal{M} \rightarrow \mathcal{N}$ such that

$$
\rho_{\theta} \circ \alpha=\rho_{\theta}, \quad \text { for all } \theta \in \Theta \text {. }
$$

If the map $\alpha$ above is two-positive, then $\mathcal{N}$ is said to be strongly sufficient, and if it is a conditional expectation onto $\mathcal{N}$, then $\mathcal{N}$ is said to be sufficient in Umegaki's sense. It can be shown that for finite-dimensional von Neumann algebras and families of faithful states sufficiency and strong sufficiency are equivalent. The notion of sufficiency in Umegaki's sense appeared in [13,14], while strong sufficiency was introduced in [4]. If the algebra $\mathcal{N}$ is sufficient and contained in any other sufficient (sufficient in strong or Umegaki's sense) algebra, then $\mathcal{N}$ is said to be minimal. It is clear that a minimal sufficient (in any sense) subalgebra is unique (if it exists). On account of [6, Theorem 1], for a faithful family of normal states on $\mathcal{M}$ there exists the minimal sufficient von Neumann subalgebra $\mathcal{M}_{\min }$ of $\mathcal{M}$.

\section{Sufficiency in finite von Neumann algebras}

In this section, we show that for a $\sigma$-finite finite von Neumann algebra $\mathcal{M}$ and a faithful family of normal states on $\mathcal{M}$ the minimal sufficient von Neumann subalgebra of $\mathcal{M}$ is sufficient in Umegaki's sense. Moreover, we also obtain a version of the factorization theorem.

In what follows, we shall need a few basic facts from modular theory of von Neumann algebras; however, this theory will be employed only to a limited extent. For its full account, the reader is referred to [10]. Let $\varphi$ be a normal faithful state on $\mathcal{M}$. There exists a group of automorphisms of $\mathcal{M},\left\{\sigma_{t}^{\varphi}: t \in \mathbb{R}\right\}$, called the modular automorphism group, associated with $\varphi$ in a canonical way. The algebra of fixed points of the modular automorphism group, denoted by $\mathcal{N}^{\varphi}$, is called the centralizer of $\varphi$, and the following relation holds

$$
\mathcal{M}^{\varphi}=\{A \in \mathcal{M}: \text { for each } B \in \mathcal{M}, \varphi(A B)=\varphi(B A)\}
$$

Let $\mathcal{N}$ be a von Neumann subalgebra of $\mathcal{M}$. If $\sigma_{t}^{\varphi}(\mathcal{N})=\mathcal{N}$ for all $t \in \mathbb{R}$, then there is a normal faithful conditional expectation $\mathbb{F}$ from $\mathcal{M}$ onto $\mathcal{N}$ such that

$$
\varphi \circ \mathbb{F}=\varphi
$$

Let $\mathcal{A}$ be a subspace of $\mathcal{M}$ containing the identity operator $\mathbb{1}$. $\mathcal{A}$ is said to be a $\mathrm{JW}^{*}$ subalgebra of $\mathcal{M}$ if it is $\sigma$-weakly closed and closed with respect to the operation of taking adjoints and the Jordan product. Then the self-adjoint part of $\mathcal{A}$ is a JW-algebra, in particular, it is generated by projections (cf. [3]). Let $\mathbb{E}$ be a normal positive faithful unital projection from the JW-algebra $\mathcal{M}_{h}$ onto a JW-algebra $\mathcal{A}$. On account of [3, 
Lemma 4.4.13], we have

$$
\mathbb{E}(A \circ B)=A \circ \mathbb{E}(B)
$$

for all $A \in \mathcal{A}_{h}, B \in \mathcal{M}_{h}$. Let now $\mathbb{E}$ be a normal positive faithful unital projection from $\mathcal{M}$ onto a $\mathrm{JW}^{*}$-algebra $\mathcal{A}$. Then $\mathbb{E} \mid \mathcal{M}_{h}$ is as above, and hence it satisfies Eq. (2). Decomposing arbitrary $A \in \mathcal{A}$ and $B \in \mathcal{M}$ as $A=A_{1}+i A_{2}, B=B_{1}+i B_{2}$ where $A_{1}, A_{2} \in \mathcal{A}_{h}, B_{1}, B_{2} \in \mathcal{M}_{h}$, we obtain the equality

$$
\mathbb{E}(A \circ B)=A \circ \mathbb{E}(B)
$$

for all $A \in \mathcal{A}, B \in \mathcal{M}$. Using the formula

$$
A B A=2 A \circ(A \circ B)-A^{2} \circ B, \quad A \in \mathcal{A}, B \in \mathcal{M},
$$

we get

$$
\mathbb{E}(S B S)=S \mathbb{E}(B) S
$$

for each $B \in \mathcal{M}$ and each symmetry $S \in \mathcal{A}_{h}$ (symmetry means that $S^{2}=\mathbb{1}$ ).

The lemma below is a $\mathrm{JW}^{*}$-version of a known result on uniqueness of conditional expectation for von Neumann algebras.

Lemma 1 Let $\varphi$ be a normal faithful state on $\mathcal{M}$, let $\mathcal{A}$ be a $J W^{*}$-subalgebra of $\mathcal{M}$, and let $\mathbb{E}_{i}: \mathcal{M} \rightarrow \mathcal{A}, i=1,2$, be normal positive unital projections onto $\mathcal{A}$ such that

$$
\varphi \circ \mathbb{E}_{1}=\varphi \circ \mathbb{E}_{2}
$$

Then $\mathbb{E}_{1}=\mathbb{E}_{2}$.

Proof Observe first that the family $\left\{\varphi_{H}: H \in \mathcal{A}\right\}$ of normal linear functionals on $\mathcal{M}$ defined as

$$
\varphi_{H}(B)=\varphi(H \circ B), \quad B \in \mathcal{M},
$$

separates the points of $\mathcal{A}$. Indeed, if we have $\varphi_{H}(A)=0$ for some $A \in \mathcal{A}$ and all $H \in \mathcal{A}$, then taking $H=A^{*}$ we get

$$
0=\varphi_{A^{*}}(A)=\frac{1}{2} \varphi\left(A^{*} A+A A^{*}\right)
$$

and the faithfulness of $\varphi$ yields $A^{*} A=0$, i.e., $A=0$. Now for arbitrary $B \in \mathcal{M}$, we have by virtue of the equality (3)

$$
\varphi_{H}\left(\mathbb{E}_{1}(B)\right)=\varphi\left(H \circ \mathbb{E}_{1}(B)\right)=\varphi\left(\mathbb{E}_{1}(H \circ B)\right)=\varphi(H \circ B)=\varphi_{H}(B),
$$


and analogously

$$
\varphi_{H}\left(\mathbb{E}_{2}(B)\right)=\varphi_{H}(B),
$$

showing that $\varphi_{H}\left(\mathbb{E}_{1}(B)\right)=\varphi_{H}\left(\mathbb{E}_{2}(B)\right)$, hence $\mathbb{E}_{1}(B)=\mathbb{E}_{2}(B)$.

Now we are ready to prove that for a faithful family of normal states the minimal sufficient subalgebra is also sufficient in Umegaki's sense.

Theorem 2 Let $\left\{\rho_{\theta}: \theta \in \Theta\right\}$ be a faithful family of normal states on a $\sigma$-finite finite von Neumann algebra $\mathcal{M}$ with a normal faithful tracial state $\tau$. The minimal sufficient von Neumann subalgebra $\mathcal{M}_{\text {min }}$ of $\mathcal{M}$ is sufficient in Umegaki's sense.

Proof As in the proof of Theorem 1 in [6], we let $\mathfrak{S}$ be the family of all normal positive linear unital maps on $\mathcal{M}$ such that the states $\rho_{\theta}$ are invariant with respect to the maps from $\mathfrak{S}$. It is seen that $\mathfrak{S}$ is a non-empty (because it contains the identity map) semigroup. Let $\mathcal{A}$ be the set of fixed points of the maps from $\mathfrak{S}$, i.e.,

$$
\mathcal{A}=\{A \in \mathcal{M}: \alpha(A)=A \text { for all } \alpha \in \mathfrak{S}\}
$$

Then $\mathcal{A}$ is a $\mathrm{JW}^{*}$-algebra, and on account of [6, Theorem 1] we have

$$
\mathcal{M}_{\min }=W^{*}(\mathcal{A})=W^{*}\left(\mathcal{A}_{h}\right)
$$

moreover, there is a normal positive faithful unital projection $\mathbb{E}$ from $\mathcal{M}$ onto $\mathcal{A}$ such that for all $\theta \in \Theta$

$$
\rho_{\theta} \circ \mathbb{E}=\rho_{\theta} .
$$

Put

$$
\varphi=\tau \circ \mathbb{E} .
$$

Then $\varphi$ is a normal state on $\mathcal{M}$ such that

$$
\varphi \circ \mathbb{E}=\varphi
$$

Let $S$ be an arbitrary symmetry in $\mathcal{A}_{h}$. Taking $B S$ instead of $B$ in the formula (4), we get

$$
\mathbb{E}(S B)=S \mathbb{E}(B S) S,
$$

hence

$$
\varphi(S B)=\tau(\mathbb{E}(S B))=\tau(S \mathbb{E}(B S) S)=\tau(\mathbb{E}(B S))=\varphi(B S) .
$$


An arbitrary projection $P$ in $\mathcal{A}_{h}$ has the form $P=\frac{1}{2}(S+\mathbb{1})$ where $S=2 P-\mathbb{1}$ is a symmetry, thus

$$
\varphi(P B)=\varphi(B P)
$$

for every such projection. Since $\mathcal{A}_{h}$ is a JW-algebra, linear combinations of its projections are $\sigma$-weakly dense in $\mathcal{A}_{h}$ (cf. [3]) and the $\sigma$-weak continuity of $\varphi$ yields

$$
\varphi(A B)=\varphi(B A)
$$

for each $A \in \mathcal{A}_{h}$. Since $B \in \mathcal{M}$ was arbitrary, this means that $A \in \mathcal{N}^{\varphi}$, thus

$$
\mathcal{A}_{h} \subset \mathcal{M}^{\varphi} .
$$

Since $\mathcal{M}^{\varphi}$ is a von Neumann algebra, this yields

$$
\mathcal{M}_{\text {min }}=W^{*}\left(\mathcal{A}_{h}\right) \subset \mathcal{M}^{\varphi} .
$$

(The reasoning above follows the proof of Haagerup and Størmer [2, Lemma 2.2].) Since for the modular automorphism group we have

$$
\sigma_{t}^{\varphi}(A)=A \text { for all } A \in \mathcal{M}^{\varphi}
$$

we get $\sigma_{t}^{\varphi}\left(\mathcal{M}_{\min }\right)=\mathcal{M}_{\text {min }}$ for all $t \in \mathbb{R}$, thus there is a normal faithful conditional expectation $\mathbb{F}$ from $\mathcal{M}$ onto $\mathcal{M}_{\min }$ such that

$$
\varphi \circ \mathbb{F}=\varphi
$$

We have

$$
(\mathbb{E} \circ \mathbb{F})^{2}=\mathbb{E} \circ(\mathbb{F} \circ \mathbb{E}) \circ \mathbb{F}=\mathbb{E} \circ \mathbb{E} \circ \mathbb{F}=\mathbb{E} \circ \mathbb{F}
$$

showing that $\mathbb{E} \circ \mathbb{F}$ is a normal positive unital projection onto $\mathcal{A}$. Moreover,

$$
\varphi \circ(\mathbb{E} \circ \mathbb{F})=(\tau \circ \mathbb{E}) \circ(\mathbb{E} \circ \mathbb{F})=(\tau \circ \mathbb{E}) \circ \mathbb{F}=\varphi \circ \mathbb{F}=\varphi=\varphi \circ \mathbb{E},
$$

and Lemma 1 yields

$\mathbb{E} \circ \mathbb{F}=\mathbb{E}$.

Thus for each $\theta \in \Theta$, we have

$$
\rho_{\theta} \circ \mathbb{F}=\left(\rho_{\theta} \circ \mathbb{E}\right) \circ \mathbb{F}=\rho_{\theta} \circ(\mathbb{E} \circ \mathbb{F})=\rho_{\theta} \circ \mathbb{E}=\rho_{\theta},
$$

which means that $\mathcal{M}_{\min }$ is sufficient in Umegaki's sense. 
An important factorization theorem was formulated in [4, Theorem 4] in the case when the algebra $\mathcal{M}$ is a countable direct sum of type I factors. However, as it was shown in [6, Examples 2 and 3], its setup needs substantial corrections, and we shall see that this theorem holds in full generality in finite von Neumann algebras. For this purpose, let us introduce some necessary notions. Since the proof of this theorem is in its main part a repetition of the proof in [4, Section 4] with some additional reasoning, we shall follow closely the notation employed there. However, we shall use some parts of the theory of measurable operators and noncommutative $L^{p}$-spaces (actually only $L^{1}$-space) for a brief account of which we refer the reader to the "Appendix".

For a linear operator $A$ on a Hilbert space $\mathcal{H}$, by $\mathcal{D}(A)$, we denote the domain of $A$, and by $\bar{A}$ - the closure of $A$.

Let $\mathcal{M}$ be a finite von Neumann algebra with a normal faithful finite trace $\tau$, let $\mathcal{M}_{0}$ be a von Neumann subalgebra of $\mathcal{M}$, and let $\mathcal{M}_{1}=\mathcal{M}_{0}^{\prime} \cap \mathcal{M}$ be the relative commutant of $\mathcal{M}_{0}$. By $\tilde{\mathcal{N}}$ we denote the algebra of measurable operators, and by $L^{1}(\mathcal{M}, \tau), L^{1}\left(\mathcal{M}_{0}, \tau\right), L^{1}\left(\mathcal{M}_{1}, \tau\right)$ the corresponding $L^{1}$-spaces. (Here we make use of the assumption that $\mathcal{M}$ is finite, otherwise there is no guarantee that $\tau \mid \mathcal{M}_{0}$ or $\tau \mid \mathcal{M}_{1}$ is semifinite.) Let $\left\{\rho_{\theta}: \theta \in \Theta\right\}$ be a faithful family of normal states on $\mathcal{M}$, and let, as before, for some convex combination

$$
\omega=\sum_{k=1}^{\infty} \lambda_{k} \rho_{\theta_{k}},
$$

$\omega$ be a faithful state on $\mathcal{M}$. Denote

$$
\omega_{0}=\omega\left|\mathcal{M}_{0}, \quad \omega_{1}=\omega\right| \mathcal{M}_{1}, \quad \tau_{0}=\tau\left|\mathcal{M}_{0}, \quad \tau_{1}=\tau\right| \mathcal{M}_{1},
$$

and let $D_{\omega} \in L^{1}(\mathcal{M}, \tau), D_{\omega_{0}} \in L^{1}\left(\mathcal{M}_{0}, \tau_{0}\right), D_{\omega_{1}} \in L^{1}\left(\mathcal{M}_{1}, \tau_{1}\right)$ be the densities of $\omega, \omega_{0}$ and $\omega_{1}$, respectively. Now the reasoning of [4, p. 269] can be repeated word for word upon observing that the modular automorphism groups $\left\{\sigma_{t}^{\omega}\right\},\left\{\sigma_{t}^{\omega_{0}}\right\}$ and $\left\{\sigma_{t}^{\omega_{1}}\right\}$ have the same form as in [4], i.e., for instance

$$
\sigma_{t}^{\omega}(A)=D_{\omega}^{i t} A D_{\omega}^{-i t}, \quad A \in \mathcal{M}
$$

and similarly for the remaining two (cf. [10, Proposition 4.7]). This leads to the equality

$$
D_{\omega}^{i t}=D_{\omega_{0}}^{i t} D_{\omega_{1}}^{i t} z^{i t}, \quad t \in \mathbb{R},
$$

for some positive self-adjoint $z$ with trivial null-space, affiliated with the centre of $\mathcal{M}_{1}$.

Denote by $D_{\theta}, D_{\theta, 0}$ the densities of the states $\rho_{\theta}$ and $\rho_{\theta} \mid \mathcal{M}_{0}$, respectively. Now the factorization theorem reads

Theorem 3 The subalgebra $\mathcal{M}_{0}$ is sufficient for a faithful family of normal states $\left\{\rho_{\theta}: \theta \in \Theta\right\}$ if and only if

$$
D_{\theta}=\overline{D_{\theta, 0} D_{\omega_{1}} z}
$$


where $z$ is a positive self-adjoint operator with trivial null-space, affiliated with the centre of $\mathcal{M}_{1}$.

Proof Fix $\theta$, and let

$$
\begin{aligned}
D_{\theta, 0} & =\int_{0}^{\infty} \lambda E_{0}(\mathrm{~d} \lambda), \\
D_{\omega_{1}} & =\int_{0}^{\infty} \lambda E_{1}(\mathrm{~d} \lambda), \\
z & =\int_{0}^{\infty} \lambda E_{z}(\mathrm{~d} \lambda),
\end{aligned}
$$

be the spectral decompositions of $D_{\theta, 0}, D_{\omega_{1}}$ and $z$. Since $D_{\theta, 0}$ is affiliated with $\mathcal{M}_{0}$, $D_{\omega_{1}}$ is affiliated with $\mathcal{M}_{1}$, and $z$ is affiliated with the centre of $\mathcal{M}_{1}$, it follows that the spectral measures $E_{0}, E_{1}$ and $E_{z}$ take values in $\mathcal{M}_{0}, \mathcal{M}_{1}$ and the centre of $\mathcal{M}_{1}$, respectively, thus they mutually commute. Consequently, there exists a spectral measure $E$ in $\mathcal{M}$, and nonnegative Borel functions $g_{0}, g_{1}$ and $h$ such that

$$
\begin{aligned}
D_{\theta, 0} & =\int_{-\infty}^{\infty} g_{0}(\lambda) E(\mathrm{~d} \lambda), \\
D_{\omega_{1}} & =\int_{-\infty}^{\infty} g_{1}(\lambda) E(\mathrm{~d} \lambda), \\
z & =\int_{-\infty}^{\infty} h(\lambda) E(\mathrm{~d} \lambda) .
\end{aligned}
$$

From these equalities, we obtain

$$
D_{\theta, 0} D_{\omega_{1}} z \subset \int_{-\infty}^{\infty} g_{0}(\lambda) g_{1}(\lambda) h(\lambda) E(\mathrm{~d} \lambda):=B .
$$

Operator $B$ on the right hand side of the above relation is a self-adjoint operator affiliated with $\mathcal{M}$, i.e., it belongs to the algebra $\tilde{\mathcal{M}}$ of measurable operators, while on the left hand side we have a product of operators from $\widetilde{\mathcal{M}}$. From the properties of $\widetilde{\mathcal{M}}$, it follows that

$$
\overline{D_{\theta, 0} D_{\omega_{1}} z}=B
$$

Moreover, we have

$$
\begin{aligned}
B^{i t} & =\left[\int_{-\infty}^{\infty} g_{0}(\lambda) g_{1}(\lambda) h(\lambda) E(\mathrm{~d} \lambda)\right]^{i t} \\
& =\int_{-\infty}^{\infty} g_{0}(\lambda)^{i t} g_{1}(\lambda)^{i t} h(\lambda)^{i t} E(\mathrm{~d} \lambda)=D_{\theta, 0}^{i t} D_{\omega_{1}}^{i t} z^{i t} .
\end{aligned}
$$


Now the proof follows the lines of the proof of Theorem 4 in [4]. The crucial observation is that also in the case of, in general unbounded, operators $D_{\theta}, D_{\omega}, D_{\theta, 0}$ and $D_{\omega_{0}}$ the following equalities for the Connes cocycles hold true

$$
\begin{aligned}
{\left[D \rho_{\theta}: D \omega\right]_{t} } & =\left[D \rho_{\theta}: D \tau\right]_{t}[D \tau: D \omega]_{t} \\
& =\left[D \rho_{\theta}: D \tau\right]_{t}[D \omega: D \tau]_{t}^{-1}=D_{\theta}^{i t} D_{\omega}^{-i t},
\end{aligned}
$$

(cf. Araki and Masuda [1, Theorem C.1] for the chain rule in the first equality above, Strătilă [10, Corollary 3.4] for the second equality, and Strătilă [10, Corollary 4.8] for the third). Similarly,

$$
\left[D \rho_{\theta, 0}: D \omega_{0}\right]_{t}=D_{\theta, 0}^{i t} D_{\omega_{0}}^{-i t} .
$$

Assume that $\mathcal{M}_{0}$ is sufficient. Then on account of [4, Theorem 1], we have [ $D \rho_{\theta}$ : $\left.D_{\omega}\right]_{t}=\left[D \rho_{\theta, 0}: D_{\omega_{0}}\right]_{t}$, thus from (9), (10) and the fact that $D_{\omega}^{i t}$ are unitary, we get

$$
D_{\theta}^{i t}=D_{\theta, 0}^{i t} D_{\omega_{0}}^{-i t} D_{\omega}^{i t}
$$

Using (6) together with the fact that $D_{\omega_{0}}^{i t}$ are also unitary, we obtain

$$
D_{\theta}^{i t}=D_{\theta, 0}^{i t} D_{\omega_{0}}^{-i t} D_{\omega_{0}}^{i t} D_{\omega_{1}}^{i t} z^{i t}=D_{\theta, 0}^{i t} D_{\omega_{1}}^{i t} z^{i t}=B^{i t}
$$

From this easily follows that $D_{\theta}=B$. Indeed, denoting by $\mathcal{N}(T)$ the null-space of the operator $T$, we have from the spectral theorem

$$
\mathcal{N}\left(D_{\theta}^{i t}\right)=\mathcal{N}\left(D_{\theta}\right) \quad \text { and } \quad \mathcal{N}\left(B^{i t}\right)=\mathcal{N}(B)
$$

so

$$
\mathcal{N}\left(D_{\theta}\right)=\mathcal{N}\left(D_{\theta}^{i t}\right)=\mathcal{N}\left(B^{i t}\right)=\mathcal{N}(B)
$$

Denote by $P$ the projection onto the common null-space above, and let

$$
D_{\theta}=\int_{0}^{\infty} \lambda E^{\prime}(\mathrm{d} \lambda)
$$

be the spectral decomposition of $D_{\theta}$. Then

$$
P+D_{\theta}=\int_{0}^{\infty}\left[\chi_{\{0\}}(\lambda)+\lambda\right] E^{\prime}(\mathrm{d} \lambda),
$$


where $\chi_{\{0\}}$ denotes the indicator function of the set $\{0\}$. Hence

$$
\begin{aligned}
\left(P+D_{\theta}\right)^{i t} & =\int_{0}^{\infty}\left[\chi_{\{0\}}(\lambda)+\lambda\right]^{i t} E^{\prime}(\mathrm{d} \lambda)=\int_{0}^{\infty}\left[\chi_{\{0\}}(\lambda)+\lambda^{i t}\right] E^{\prime}(\mathrm{d} \lambda) \\
& =P+\int_{0}^{\infty} \lambda^{i t} E^{\prime}(\mathrm{d} \lambda)=P+D_{\theta}^{i t}
\end{aligned}
$$

and similarly

$$
(P+B)^{i t}=P+B^{i t},
$$

which on account of the equality (11) yields

$$
\left(P+D_{\theta}\right)^{i t}=(P+B)^{i t}
$$

But the operators $P+D_{\theta}$ and $P+B$ have trivial null-spaces, which means that the equality above is the identity of two unitary groups. It follows that their generators must be the same, so

$$
P+D_{\theta}=P+B
$$

i.e.,

$$
D_{\theta}=B,
$$

and the equality (8) proves the claim.

Assume now that the equality (7) holds. In terms of the algebra $\tilde{\mathcal{M}}$, it can be rewritten as

$$
D_{\theta}=D_{\theta, 0} \cdot D_{\omega_{1}} \cdot z
$$

(where the central dot stands for the strong multiplication-see the "Appendix"). Denote

$$
\omega_{n}=\sum_{k=1}^{n} \lambda_{k} \rho_{\theta_{k}} .
$$

Then by virtue of the isomorphism between $\mathcal{M}_{*}$ and $L^{1}(\mathcal{M}, \tau)$, we have

$$
D_{\omega_{n}}=s-\sum_{k=1}^{n} \lambda_{k} D_{\theta_{k}},
$$


where $s-\sum$ denotes the strong sum. The same holds of course for $\omega_{n, 0}=\omega_{n} \mid \mathcal{M}_{0}$, i.e.,

$$
D_{\omega_{n, 0}}=s-\sum_{k=1}^{n} \lambda_{k} D_{\theta_{k, 0}},
$$

so from the formula (7) and the fact that $\tilde{\mathcal{M}}$ is an algebra, we obtain

$$
\begin{aligned}
D_{\omega_{n}} & =s-\sum_{k=1}^{n} \lambda_{k} D_{\theta_{k}}=s-\sum_{k=1}^{n} \lambda_{k} D_{\theta_{k, 0}} \cdot D_{\omega_{1}} \cdot z \\
& =D_{\omega_{n, 0}} \cdot D_{\omega_{1}} \cdot z .
\end{aligned}
$$

Since $\omega_{n} \rightarrow \omega$ in norm in $\mathcal{M}_{*}$, we infer that $D_{\omega_{n}} \rightarrow D_{\omega}$ in the norm $\|\cdot\|_{1}$, and by the same token $D_{\omega_{n, 0}} \rightarrow D_{\omega_{0}}$. According to Lemma 5 (see the Appendix), we have $D_{\omega_{n}} \rightarrow D_{\omega}$ and $D_{\omega_{n, 0}} \rightarrow D_{\omega_{0}}$ in measure. Since $\tilde{\mathcal{M}}$ is a topological *-algebra with respect to the measure topology, the formula (12) yields

$$
D_{\omega}=D_{\omega_{0}} \cdot D_{\omega_{1}} \cdot z
$$

in other words

$$
D_{\omega}=\overline{D_{\omega_{0}} D_{\omega_{1}} z}
$$

Now we proceed as in the first part of the proof. Since the operators $D_{\omega_{0}}, D_{\omega_{1}}$ and $z$ commute, from the equalities (7) and (13), we obtain

$$
D_{\theta}^{i t}=D_{\theta, 0}^{i t} D_{\omega_{1}}^{i t} z^{i t}, \quad D_{\omega}^{i t}=D_{\omega_{0}}^{i t} D_{\omega_{1}}^{i t} z^{i t} .
$$

Thus on account of commutation properties and the fact that $\left\{D_{\omega_{1}}^{i t}\right\}$ and $\left\{z^{i t}\right\}$ are unitary groups, we get

$$
\begin{aligned}
{\left[D \rho_{\theta}: D \omega\right]_{t} } & =D_{\theta}^{i t} D_{\omega}^{-i t}=D_{\theta, 0}^{i t} D_{\omega_{1}}^{i t} z^{i t} D_{\omega_{0}}^{-i t} D_{\omega_{1}}^{-i t} z^{-i t} \\
& =D_{\theta, 0}^{i t} D_{\omega_{0}}^{-i t}=\left[D \rho_{\theta, 0}: D \omega_{0}\right]_{t},
\end{aligned}
$$

which by virtue of [4, Theorem 1] yields the sufficiency of $\mathcal{M}_{0}$.

\section{Sufficiency for pure states on $\mathbb{B}(\mathcal{H})$}

In this section, we shall be dealing with the full algebra $\mathcal{M}=\mathbb{B}(\mathcal{H})$ and pure $(\equiv$ vector) states. Let $\left\{\psi_{\theta}: \theta \in \Theta\right\}$ be a faithful family of pure states. According to [6, Theorem 1], there exists the minimal sufficient for this family subalgebra of $\mathbb{B}(\mathcal{H})$. It turns out that this algebra admits a straightforward description. 
Theorem 4 Let $\left\{\psi_{\theta}: \theta \in \Theta\right\}$ be a faithful family of pure states on $\mathbb{B}(\mathcal{H})$, and let $\mathcal{M}_{\min }$ be the minimal sufficient for this family subalgebra of $\mathbb{B}(\mathcal{H})$. Then

(i) $\mathcal{M}_{\min }$ is generated by $P_{\left[\psi_{\theta}\right]}$, i.e., $\mathcal{M}_{\min }=W^{*}\left(\left\{P_{\left[\psi_{\theta}\right]}: \theta \in \Theta\right\}\right)$.

(ii) There exists a decomposition

$$
\mathcal{H}=\bigoplus_{i} \mathcal{H}_{i}
$$

such that

$$
\mathcal{M}_{\min }=\sum_{i}^{\oplus} \mathbb{B}\left(\mathcal{H}_{i}\right)
$$

Proof First observe that $\mathrm{s}\left(\psi_{\theta}\right)=P_{\left[\psi_{\theta}\right]}$. As in Theorem 2, denote by $\mathfrak{S}$ the family of all normal positive unital maps on $\mathcal{N}$ such that the states $\psi_{\theta}$ are invariant with respect to the maps from $\mathfrak{S}$. On account of [12, Theorem 4], for every $\theta \in \Theta$, we have $\alpha\left(P_{\left[\psi_{\theta}\right]}\right)=P_{\left[\psi_{\theta}\right]}$ for each $\alpha \in \mathfrak{S}$, which by virtue of [6, Theorem 1] yields the relation $P_{\left[\psi_{\theta}\right]} \in \mathcal{M}_{\min }$.

For the sake of brevity, set $\mathcal{R}=\left\{\psi_{\theta}: \theta \in \Theta\right\}$, and define on $\mathcal{R}$ an equivalence relation as follows: $\psi_{\theta^{\prime}} \equiv \psi_{\theta^{\prime \prime}}$ if there exists a finite string $\psi_{\theta_{1}}, \ldots, \psi_{\theta_{m}}$ such that $\psi_{\theta_{1}}=\psi_{\theta^{\prime}}, \psi_{\theta_{m}}=\psi_{\theta^{\prime \prime}}$ and

$$
\left\langle\psi_{\theta_{j}} \mid \psi_{\theta_{j+1}}\right\rangle \neq 0 \quad \text { for } \quad j=1, \ldots, m-1
$$

Let

$$
\mathcal{R}=\bigcup_{i} \mathcal{R}_{i}
$$

be the partition of $\mathcal{R}$ determined by the relation $\equiv$. Denote $\mathcal{R}_{i}=\left\{\psi_{\theta}: \theta \in \Theta_{i}\right\}$. It is obvious that for all $i^{\prime}, i^{\prime \prime}$ such that $i^{\prime} \neq i^{\prime \prime}$, we have $\mathcal{R}_{i^{\prime}} \perp \mathcal{R}_{i^{\prime \prime}}$. Put

$$
P_{i}=\bigvee_{\theta \in \Theta_{i}} P_{\left[\psi_{\theta}\right]} .
$$

It follows that $P_{i}$ are pairwise orthogonal, and the faithfulness of $\left\{\psi_{\theta}: \theta \in \Theta\right\}$ yields

$$
\sum_{i} P_{i}=\mathbb{1}
$$

Denote $\mathcal{H}_{i}=P_{i}(\mathcal{H})$. Then

$$
\mathcal{H}=\bigoplus_{i} \mathcal{H}_{i}
$$

Fix arbitrary $i$ and consider the family of pure states $\mathcal{R}_{i}=\left\{\psi_{\theta}: \theta \in \Theta_{i}\right\}$ on the Hilbert space $\mathcal{H}_{i}$. Take arbitrary $\psi_{\theta^{\prime}}, \psi_{\theta^{\prime \prime}} \in \mathcal{R}_{i}$, and let $\psi_{\theta_{1}}, \ldots, \psi_{\theta_{m}}$, with $\psi_{\theta_{1}}=\psi_{\theta^{\prime}}$ 
and $\psi_{\theta_{m}}=\psi_{\theta^{\prime \prime}}$ be the string as in the definition of the equivalence relation $\equiv$. From this definition, it follows that $\psi_{\theta_{j}} \equiv \psi_{\theta_{k}}$ for all $j, k=1, \ldots, m$, thus $\psi_{\theta_{j}} \in \mathcal{R}_{i}$ for all $j=1, \ldots, m$. Consequently, $P_{\left[\psi_{\theta_{j}}\right]} \leq P_{i}$, so we can consider the projections $P_{\left[\psi_{\theta_{j}}\right]}$ on the Hilbert space $\mathcal{H}_{i}$. We have

$$
P_{\left[\psi_{\theta_{1}}\right]} \ldots P_{\left[\psi_{\theta_{m}}\right]}=a E_{\psi_{\theta_{1}}, \psi_{\theta_{m}}}=a E_{\psi_{\theta^{\prime}}, \psi_{\theta^{\prime \prime}}}
$$

where

$$
a=\left\langle\psi_{\theta_{1}} \mid \psi_{\theta_{2}}\right\rangle \cdots\left\langle\psi_{\theta_{m-1}} \mid \psi_{\theta_{m}}\right\rangle \neq 0
$$

and for $\xi, \eta \in \mathcal{H}$ the operator $E_{\xi, \eta}$ is defined as

$$
E_{\xi, \eta} \zeta=\langle\eta \mid \zeta\rangle \xi, \quad \zeta \in \mathcal{H}
$$

(The formula (15) is obvious when using Dirac notation since then on the left hand side we have simply

$$
\left|\psi_{\theta_{1}}\right\rangle\left\langle\psi_{\theta_{1}} \mid \psi_{\theta_{2}}\right\rangle \cdots\left\langle\psi_{\theta_{m-1}} \mid \psi_{\theta_{m}}\right\rangle\left\langle\psi_{\theta_{m}}\left|=\left\langle\psi_{\theta_{1}} \mid \psi_{\theta_{2}}\right\rangle \cdots\left\langle\psi_{\theta_{m-1}} \mid \psi_{\theta_{m}}\right\rangle\right| \psi_{\theta_{1}}\right\rangle\left\langle\psi_{\theta_{m}}\right|,
$$

which is just the right-hand side. In this notation, we have $E_{\xi, \eta}=|\xi\rangle\langle\eta|$.)

Denote $\mathcal{N}=W^{*}\left(\left\{P_{\left[\psi_{\theta}\right]}: \theta \in \Theta\right\}\right)$. On account of the above considerations, we infer that $E_{\psi_{\theta^{\prime}}, \psi_{\theta^{\prime \prime}}} \in \mathcal{N}$ for all $\psi_{\theta^{\prime}}, \psi_{\theta^{\prime \prime}} \in \mathcal{R}_{i}$.

Let

$$
0 \neq \xi=\sum_{j=1}^{m} a_{j} \psi_{\theta_{j}}, \quad \psi_{\theta_{j}} \in \mathcal{R}_{i}
$$

be an arbitrary finite linear combination of vectors from $\mathcal{R}_{i}$. We have

$$
P_{[\xi]}=\frac{1}{\|\xi\|^{2}} \sum_{j, k=1}^{m} a_{j} \bar{a}_{k} E_{\psi_{\theta_{j}}, \psi_{\theta_{k}}}
$$

(This is again most easily seen by using Dirac notation since then

$$
\left.|\xi\rangle\left\langle\xi|=| \sum_{j=1}^{m} a_{j} \psi_{\theta_{j}}\right\rangle\left\langle\sum_{k=1}^{m} a_{k} \psi_{\theta_{k}}\left|=\sum_{j, k=1}^{m} a_{j} \bar{a}_{k}\right| \psi_{\theta_{j}}\right\rangle\left\langle\psi_{\theta_{k}}\right| .\right)
$$

It follows that $P_{[\xi]} \in \mathcal{N}$. Vectors $\xi$ as above lie densely in $\mathcal{H}_{i}$, so arbitrary $\eta \in \mathcal{H}_{i}$ is a limit of some $\xi_{n}$, consequently,

$$
P_{\left[\xi_{n}\right]} \rightarrow P_{[\eta]} \text { weakly, }
$$


hence $P_{[\eta]} \in \mathcal{N}$ for each $\eta \in \mathcal{H}_{i}$. Linear combinations of these projections restricted to the subspace $\mathcal{H}_{i}$ lie densely in $\mathbb{B}\left(\mathcal{H}_{i}\right)$, so for the reduced von Neumann algebra $\mathcal{N}_{P_{i}}$, we get the inclusion

$$
\mathbb{B}\left(\mathcal{H}_{i}\right) \subset \mathcal{N}_{P_{i}}
$$

and thus

$$
\mathbb{B}\left(\mathcal{H}_{i}\right)=\mathcal{N}_{P_{i}}
$$

From the definition of $P_{i}$, it follows that $P_{i}$ commutes with all $P_{\left[\psi_{\theta}\right]}, \theta \in \Theta$, because

$$
P_{i} P_{\left[\psi_{\theta}\right]}= \begin{cases}P_{\left[\psi_{\theta}\right]}, & \text { if } \theta \in \Theta_{i} \\ 0, & \text { otherwise. }\end{cases}
$$

Since the algebra $\mathcal{N}$ is generated by the projections $P_{\left[\psi_{\theta}\right]}$, we obtain that $P_{i} \in \mathcal{N}^{\prime}$. For each $A \in \mathcal{N}$, we have

$$
A=\sum_{i} A P_{i}=\sum_{i} P_{i} A
$$

showing that

$$
\mathcal{N}=\sum_{i}^{\oplus} \mathcal{N}_{P_{i}}
$$

and the formula (17) gives the representation

$$
\mathcal{N}=\sum_{i}^{\oplus} \mathbb{B}\left(\mathcal{H}_{i}\right)
$$

In the first part of the proof, we obtained the relation $P_{\left[\psi_{\theta}\right]} \in \mathcal{M}_{\min }$ for all $\theta \in \Theta$ which yields the inclusion

$$
\mathcal{N} \subset \mathcal{M}_{\min }
$$

Consider a map $\mathbb{E}: \mathbb{B}(\mathcal{H}) \rightarrow \mathcal{N}$ defined as

$$
\mathbb{E}(A)=\sum_{i} P_{i} A P_{i}
$$

It is easily seen that $\mathbb{E}$ is a normal conditional expectation such that the states $\psi_{\theta}$ are $\mathbb{E}$-invariant, thus $\mathcal{N}$ is sufficient in Umegaki's sense. Since $\mathcal{M}_{\min }$ is minimal sufficient, we obtain

$$
\mathcal{M}_{\min } \subset \mathcal{N}
$$


showing that $\mathcal{M}_{\min }=\mathcal{N}$, and the formula (18) together with the definition of $\mathcal{N}$ as $W^{*}\left(\left\{P_{\left[\psi_{\theta}\right]}: \theta \in \Theta\right\}\right)$ finish the proof.

Acknowledgements The author is indebted to the referee for valuable comments on the previous version of the paper, in particular, for pointing out an inaccuracy in the proof of Theorem 2.

\section{Declarations}

Conflict of interest There is no conflict of interest.

Open Access This article is licensed under a Creative Commons Attribution 4.0 International License, which permits use, sharing, adaptation, distribution and reproduction in any medium or format, as long as you give appropriate credit to the original author(s) and the source, provide a link to the Creative Commons licence, and indicate if changes were made. The images or other third party material in this article are included in the article's Creative Commons licence, unless indicated otherwise in a credit line to the material. If material is not included in the article's Creative Commons licence and your intended use is not permitted by statutory regulation or exceeds the permitted use, you will need to obtain permission directly from the copyright holder. To view a copy of this licence, visit http://creativecommons.org/licenses/by/4.0/.

\section{Appendix}

In this section, we collect some facts from the theory of measurable operators and noncommutative $L^{1}$-space over a von Neumann algebra with a normal faithful finite trace. A fuller account of this theory, also for a more general case of a semifinite trace and spaces $L^{p}$, can be found, e.g., in $[8,9,11,15]$.

Thus let $\mathcal{M}$ be a von Neumann algebra with a normal faithful finite trace $\tau$ acting on a Hilbert space $\mathcal{H}$. The algebra of measurable operators $\widetilde{\mathcal{N}}$ is defined as a topological *-algebra of densely defined closed operators on $\mathcal{H}$ affiliated with $\mathcal{M}$ with strong addition $\dot{+}$ and strong multiplication ·, i.e.,

$$
A \dot{+} B=\overline{A+B}, \quad A \cdot B=\overline{A B}, \quad A, B \in \tilde{\mathcal{M}},
$$

and the translation-invariant measure topology defined by a fundamental system of neighbourhoods of $0,\{N(\varepsilon, \delta): \varepsilon, \delta>0\}$, given by

$$
\begin{aligned}
& N(\varepsilon, \delta)=\{A \in \tilde{\mathcal{M}}: \text { there exists a projection } P \text { in } \mathcal{M} \text { such that } \\
& \qquad A P \in \mathcal{M}, \quad\|A P\| \leq \varepsilon \text { and } \tau(\mathbb{1}-P) \leq \delta\} .
\end{aligned}
$$

Thus for operators $A_{n}, A$ in $\tilde{\mathcal{M}}$, the sequence $\left(A_{n}\right)$ converges to $A$ in measure if for any $\varepsilon, \delta>0$ there exists $n_{0}$ such that for each $n \geq n_{0}$ there exists a projection $P \in \mathcal{M}$ such that

$$
\tau(\mathbb{1}-P) \leq \delta, \quad\left(A_{n}-A\right) P \in \mathcal{M}, \quad \text { and } \quad\left\|\left(A_{n}-A\right) P\right\| \leq \varepsilon .
$$


The following 'technical' form of convergence in measure proved in [15, Proposition 2.7] is useful. Let

$$
\left|A_{n}-A\right|=\int_{0}^{\infty} \lambda E_{n}(\mathrm{~d} \lambda)
$$

be the spectral decomposition of $\left|A_{n}-A\right|$ with spectral measures $E_{n}$ taking values in $\mathcal{M}$ since $A_{n}-A$, and thus $\left|A_{n}-A\right|$, are affiliated with $\mathcal{M}$. Then $A_{n} \rightarrow A$ in measure if and only if for each $\varepsilon>0$

$$
\tau\left(E_{n}([\varepsilon, \infty])\right) \rightarrow 0
$$

Introduce on $\mathcal{M}$ the norm $\|\cdot\|_{1}$ as follows

$$
\|A\|_{1}=\tau(|A|)
$$

The space $L^{1}(\mathcal{M}, \tau)$ is defined as the completion of $\mathcal{M}$ with respect to this norm. It turns out that $L^{1}(\mathcal{M}, \tau)$ consists of measurable operators, $\tau$ can be extended to a linear bounded positive functional on $L^{1}(\mathcal{M}, \tau)$ such that $\|A\|_{1}=\tau(|A|)$ for $A \in L^{1}(\mathcal{M}, \tau)$, and $L^{1}(\mathcal{M}, \tau)$ is isometrically isomorphic to $\mathcal{M}_{*}$ with the isomorphism given by the formula

$$
\varphi(A)=\tau(D A), \quad \varphi \in \mathcal{M}_{*}, D \in L^{1}(\mathcal{M}, \tau)
$$

The operator $D$ is called the density of $\varphi$. Moreover, if $\varphi$ is hermitian then $D=D^{*}$, and $\varphi \geq 0$ if and only if $D \geq 0$.

For an arbitrary $A \in L^{1}(\overline{\mathcal{M}}, \tau)$ we have the spectral decomposition

$$
|A|=\int_{0}^{\infty} \lambda E(\mathrm{~d} \lambda)
$$

Thus for any $\varepsilon>0$, we get

$$
|A| \geq \int_{\varepsilon}^{\infty} \lambda E(\mathrm{~d} \lambda) \geq \int_{\varepsilon}^{\infty} \varepsilon E(\mathrm{~d} \lambda)=\varepsilon E([\varepsilon, \infty]) .
$$

Consequently, we obtain the Chebyschev inequality

$$
\tau(E([\varepsilon, \infty])) \leq \frac{\tau(|A|)}{\varepsilon}=\frac{\|A\|_{1}}{\varepsilon} .
$$

Taking into account the above-mentioned 'technical' form of convergence in measure, we have

Lemma 5 If a sequence $\left(A_{n}\right)$ of operators in $L^{1}(\mathcal{M}, \tau)$ converges in $\|\cdot\|_{1}$-norm, then it converges in measure. 


\section{References}

1. Araki, H., Masuda, T.: Positive cones and $L_{p}$-spaces for von Neumann algebras. Publ. Res. Inst. Math. Sci. 18, 339-411 (1982)

2. Haagerup, U., Størmer, E.: Positive projections of von Neumann algebras onto JW-algebras. Rep. Math. Phys. 36, 317-330 (1995)

3. Hanche-Olsen, H., Størmer, E.: Jordan Operator Algebras. Pitman, Boston (1984)

4. Jenčová, A., Petz, D.: Sufficiency in quantum statistical inference. Commun. Math. Phys. 263, 259-276 (2006)

5. Jenčová, A., Petz, D.: Sufficiency in quantum statistical inference. A survey with examples. Inf. Dim. Anal. Quant. Probab. 9, 331-352 (2006)

6. Łuczak, A.: Quantum sufficiency in the operator algebra framework. Int. J. Theor. Phys. 53, 3423-3433 (2014)

7. Łuczak, A.: On a general concept of sufficiency in von Neumann algebras. Probab. Math. Stat. 35(2), 313-324 (2015)

8. Nelson, E.: Notes on non-commutative integration. J. Funct. Anal. 15, 103-116 (1974)

9. Segal, I.E.: A non-commutative extension of abstract integration. Ann. Math. 57, $401-457$ (1953)

10. Strătilă, Ş.: Modular Theory in Operator Algebras. Editura Academiei and Abacus Press, BucureştiKent (1981)

11. Takesaki, M.: Theory of Operator Algebras II. Springer, Berlin (2003)

12. Thomsen, K.E.: Invariant states for positive operator semigroups. Stud. Math. 81, 285-291 (1985)

13. Umegaki, H.: Conditional expectation in an operator algebra, III. Kōdai Math. Sem. Rep. 11, 51-64 (1959)

14. Umegaki, H.: Conditional expectation in an operator algebra, IV (entropy and information). Kōdai Math. Sem. Rep. 14, 59-85 (1962)

15. Yeadon, F.J.: Non-commutative $L_{p}$-spaces. Math. Proc. Cambr. Philos. Soc. 77, 91-102 (1975)

Publisher's Note Springer Nature remains neutral with regard to jurisdictional claims in published maps and institutional affiliations. 